\title{
HIGHEST WEIGHT MODULES FOR HERMITIAN SYMMETRIC PAIRS OF EXCEPTIONAL TYPE
}

\author{
THOMAS J. ENRIGHT AND BRAD SHELTON
}

(Communicated by Jonathan M. Rosenberg)

\begin{abstract}
We analyze the categories of highest weight modules with a semiregular generalized infinitesimal character for the two exceptional Hermitian symmetric cases. These categories are completely described, and, as a consequence, we see that the combinatorial description of the general (regular integral) categories of highest weight modules previously given in the classical cases holds also in the exceptional cases.
\end{abstract}

\section{INTRODUCTION}

In [4], the categories of highest weight modules were analyzed in the setting of classical Hermitian symmetric pairs. The main themes involved reduction from regular to semiregular generalized infinitesimal character and application of certain equivalences of categories which allowed an induction on the rank of the Lie algebra. For the exceptional cases we were not aware that the same principles apply.

More specifically, in [4] certain sets of orthogonal roots were described. It was shown for the classical Hermitian symmetric cases that these sets of roots could be used to combinatorially describe the important structure constants of the categories of highest weight modules. In particular they describe the composition factors of generalized Verma modules, the homomorphisms between generalized Verma modules, extensions between generalized Verma modules and simple modules and the Kazhdan-Lusztig-Vogan polynomials. In this paper we analyze the categories of highest weight modules with a semiregular generalized infinitesimal character for the two exceptional Hermitian symmetric cases. These categories are completely described and, as a consequence, we see that the combinatorial description of the general (regular integral) categories of highest

Received by the editors September 1, 1988 .

1980 Mathematics Subject Classification (1985 Revision). Primary 22E46, 22 E47.

Key words and phrases. Highest weight modules, Verma modules, Hermitian symmetric pairs, exceptional Lie groups.

The first author was supported in part by NSF grant DMS 86-01347 
weight modules given in [4] holds in the exceptional cases as well as in the classical cases. Many of the above described structure constants of these categories in the exceptional cases are already known (e.g. [1], [2], and [5]). However, what we present here, the description of the semiregular categories, is new, and shows that the exceptional cases are consistent with the combinatorial axioms from which the results of [4] emerge.

\section{THE CASE $\left(E_{6}, D_{5}\right)$}

We shall adhere to most of the notational conventions of [4]. Refer to that Memoir for any unexplained notation. Let $g$ denote the complex Lie algebra of type $E_{6}$ and let $\underline{p}=\underline{m} \oplus \underline{u}$ be a maximal parabolic subalgebra with $\underline{u}$ Abelian. Then $[\underline{m}, \underline{m}]$ is a simple Lie algebra of type $D_{5}$. Let $\underline{h}$ be a Cartan subalgebra of both $\underline{m}$ and $\underline{g}$. Using the notation of $\S 2$ of [4], for $\lambda \in \mathscr{P}_{\underline{m}}$ we have generalized Verma modules $N(\underline{g}, \underline{p}, \lambda)=N(\lambda)$ and categories of highest weight modules $\mathscr{O}(g, \underline{p}, \lambda)=\mathscr{O}(\lambda)$. Let $\mathscr{O}=\mathscr{O}(\rho), \rho$ the half sum of a fixed set of positive roots for $\underline{g}$. The highest weights (plus $\rho$ ) which occur in $\mathscr{O}$ correspond to the elements of $\mathscr{W}^{\underline{m}}$. This partially ordered set is given in Figure 2.1. In the figure the Dynkin diagram is labeled with simple roots $a$ through $f$. In particular, $a$ denotes the unique simple positive root of $\underline{g}$ that is not a root of $\underline{m}$ and the vertex labeled 26 denotes the identity element of the Weyl group $\mathscr{W}$. If an edge of the diagram is labeled as follows:

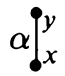

then $x<y, l(y)=l(x)-1$ and $x=y s_{\alpha}$. (Here $s_{\alpha}$ is the simple reflection corresponding to the simple root $\alpha,<$ is the Bruhat order and $l(\cdot)$ is the length function on $\mathscr{W}$.)

Recall from 9.2 of [4] the definition of $\mathscr{W}_{\alpha}$ for a simple root $\alpha$.

$$
\mathscr{W}_{\alpha}=\left\{w \in \mathscr{W}^{\underline{m}} \mid w<w s_{\alpha} \text { and } w\left(\rho-\omega_{\alpha}\right) \in \mathscr{P}_{\underline{m}}\right\} .
$$

The elements of $\mathscr{W}_{\alpha}$ correspond to the simple modules in $\mathscr{O}\left(\rho-\omega_{\alpha}\right)$. To simplify notation we put $\mathscr{O}_{\alpha}=\mathscr{O}\left(\rho-\omega_{\alpha}\right)$ for any simple root $\alpha$. It is easily seen from Figure 2.1 that for each simple root $\alpha$ the set $\mathscr{W}_{\alpha}$ consists of 6 elements and is linearly ordered by the Bruhat order. So for any $\alpha$ we will write $\mathscr{W}_{\alpha}=\left\{w_{1}, w_{2}, \ldots, w_{6}\right\}$, where $w_{1}<w_{2}<w_{3}<w_{4}<w_{5}<w_{6}$ and the exact meaning of the $w_{i}$ will depend on the $\alpha$. For example, the subset of $\mathscr{W}^{\underline{m}}$ denoted by $\mathscr{W}_{a}$ is given as (2.2) below.

Proposition 2.3. Fix a simple root $\alpha$ and for $1 \leq i \leq 6$ let $N_{i}=N\left(w_{i}\left(\rho-\omega_{\alpha}\right)\right)$ and $L_{i}=L\left(w_{i}\left(\rho-\omega_{\alpha}\right)\right)$. Then

(i) $N_{6}$ is projective and is the projective cover of $L_{6}$. For $1 \leq i \leq 5$ the projective cover of $L_{i}$ and $N_{i}$ is a nonsplit extension of $N_{i}$ by $N_{i+1}$.

(ii) $\operatorname{Ext}^{k}\left(N_{i}, L_{j}\right)= \begin{cases}\mathrm{C} & \text { if } k=j-i \\ 0 & \text { otherwise. }\end{cases}$ 

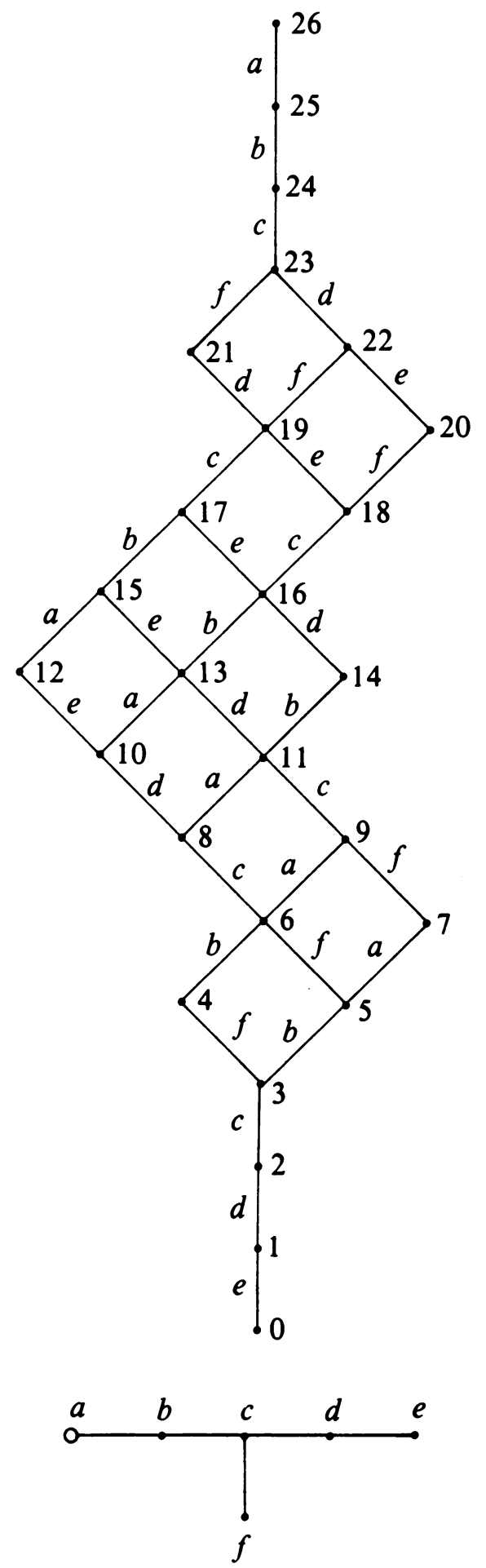

FIGURE 2.1. $\left(E_{6}, D_{5}\right)$ 


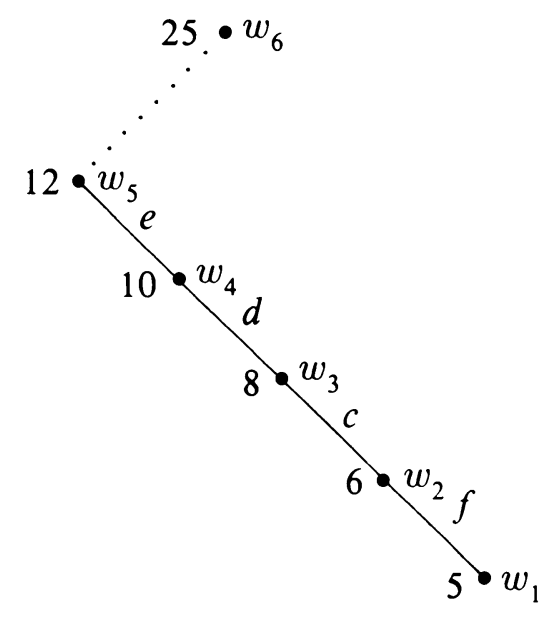

Here the dotted line indicates a Bruhat relation that is not given by right multiplication by a single simple reflection.

All of the semiregular categories $\mathscr{O}_{\alpha}$ are equivalent in this equal root length case (cf. proof of 10.1 in [4]). These equivalences are a consequence of a theorem of Vogan [6, Theorem 3.2] and correspond to pairs of adjacent simple roots (in the Dynkin diagram). Let $\tau_{\alpha \beta}$ denote this equivalence of $\mathscr{O}_{\alpha}$ onto $\mathscr{O}_{\beta}$ whenever $\alpha$ and $\beta$ are adjacent simple roots. Since $\tau_{\alpha \beta}$ sends generalized Verma modules to generalized Verma modules it induces a bijection of $\mathscr{W}_{\alpha}$ onto $\mathscr{W}_{\beta}$. It is clear that this induced map must preserve Bruhat relations, and so in our $w_{i}$ notation for $\mathscr{W}_{\alpha}$ it is the identity map.

In light of the above equivalences it suffices to prove the proposition when $\alpha$ is the simple root $a$. Moreover, the first statement of part (i) is well known and part (ii) follows from part (i) by constructing projective resolutions of the modules $N_{k}$ using the information in part (i). Let $P_{i}$ be the projective cover in $\mathscr{O}_{\alpha}$ of $L_{i}, 1 \leq i \leq 6$.

Let $\beta$ be any simple root orthogonal to $a$, i.e. $\beta \in\{c, d, e, f\}$. We see from 2.2 that $\mathscr{W}_{a} \cap \mathscr{W}_{\beta}$ has only one element, say $w_{i}$. This implies that the category $\mathscr{O}_{a, \beta}=\mathscr{O}\left(\rho-\omega_{a}-\omega_{\beta}\right)$ has a unique simple module $M$ that is projective and is a generalized Verma module. Let $\phi: \mathscr{O}_{a, \beta} \rightarrow \mathscr{O}_{a}$ be the appropriate Zuckerman translation functor. By $[3, \S 5], \phi(M)$ is the projective cover $P_{i}$ of $L_{i}$ and is a nonsplit extension of $N_{i}$ by $N_{i+1}$ (since $w_{i} s_{\beta}=w_{i+1}$ ). Refering to (2.2) we see that this paragraph proves part (i) for $i=1,2,3$, or 4 . It remains only to analyze $P_{5}$. This was the main difficulty we observed some years ago. We now offer a straightforward solution.

Set $\tau=\tau_{c f} \circ \tau_{b c} \circ \tau_{a b}$. Then $\tau: \mathscr{O}_{a} \rightarrow \mathscr{O}_{f}$ is an equivalence. Now $\mathscr{W}_{f}$ is the subset of $\mathscr{W}^{\underline{m}}$ : 


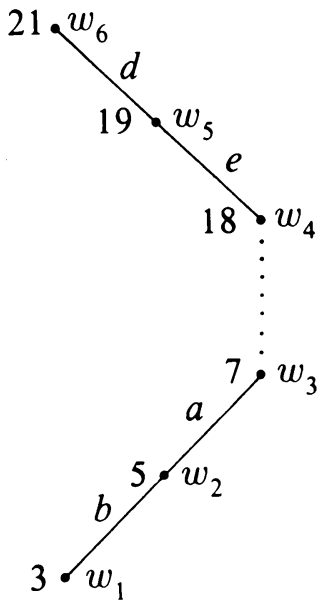

Thus $\mathscr{W}_{d} \cap \mathscr{W}_{f}=\{19\}$ and so by the argument of the preceding paragraph $\tau\left(P_{5}\right)$ is a nonsplit extension of $\tau\left(N_{5}\right)$ by $\tau\left(N_{6}\right)$. In particular, $P_{5}$ is a nonsplit extension of $N_{5}$ by $N_{6}$. This completes the proof of the proposition.

\section{THE CASE $\left(E_{7}, E_{6}\right)$}

Here we assume that $\underline{g}$ has type $E_{7}$ and $\underline{m}$ has type $E_{6}$. Otherwise let notation be as in $\S 2$ with Figure 2.1 replaced by Figure 3.1. In this section we prove that all the semiregular categories $\mathscr{O}_{\alpha}$ resemble the regular integral category for the Hermitian symmetric pair $\left(D_{6}, D_{5}\right)$. Before doing this we must analyze the categories of modules with a highest weight (plus $\rho$ ) that have an $A_{1} \times A_{1}$ singularity.

For any orthogonal simple roots $\beta$ and $\gamma$ let $\mathscr{O}_{\beta \gamma}=\mathscr{O}\left(\rho-\omega_{\beta}-\omega_{\gamma}\right)$. Put $\mathscr{W}_{\beta \gamma}=\mathscr{W}_{\beta} \cap \mathscr{W}_{\gamma}$ so that $\mathscr{W}_{\beta \gamma}$ parameterizes the simple modules in $\mathscr{O}_{\beta \gamma}$. From Figure 3.1 we see that $\mathscr{W}_{\beta \gamma}$ is always a two-element set with one element less than the other in the Bruhat order. We now prove that each of the categories $\mathscr{O}_{\beta \gamma}$ resembles the category $\mathscr{O}$ for $\operatorname{sl}(2, \mathbf{C})$.

Lemma 3.2. Let $\alpha, \beta$, and $\gamma$ be simple roots with $\alpha$ and $\beta$ adjacent and $\gamma$ orthogonal to both $\alpha$ and $\beta$. Then $\mathscr{O}_{\alpha \gamma}$ and $\mathscr{O}_{\beta \gamma}$ are equivalent categories.

Proof. Let $v$ be in $\mathscr{W}_{\alpha \gamma}$. Since $\underline{u}$ is Abelian, $v$ must occur in one of the configurations in (3.3). By the orthogonality of $\gamma$ we obtain subdiagrams of Figure 3.1 of the form in (3.4). Observe that each block in (3.4) contains a unique element of $\mathscr{W}_{\beta \gamma}$, say $w$. In the first block $w=v s_{\beta}$ and in the second block $w=v s_{\alpha}$. If $\mu$ is a composition of the translation functor from $\mathscr{O}_{\alpha \gamma}$ to $\mathscr{O}_{\gamma}$ followed by the translation functor from $\mathscr{O}_{\gamma}$ to $\mathscr{O}_{\beta \gamma}$ then $\mu\left(N\left(v\left(\rho-\omega_{\alpha}-\omega_{\gamma}\right)\right)=\right.$ 


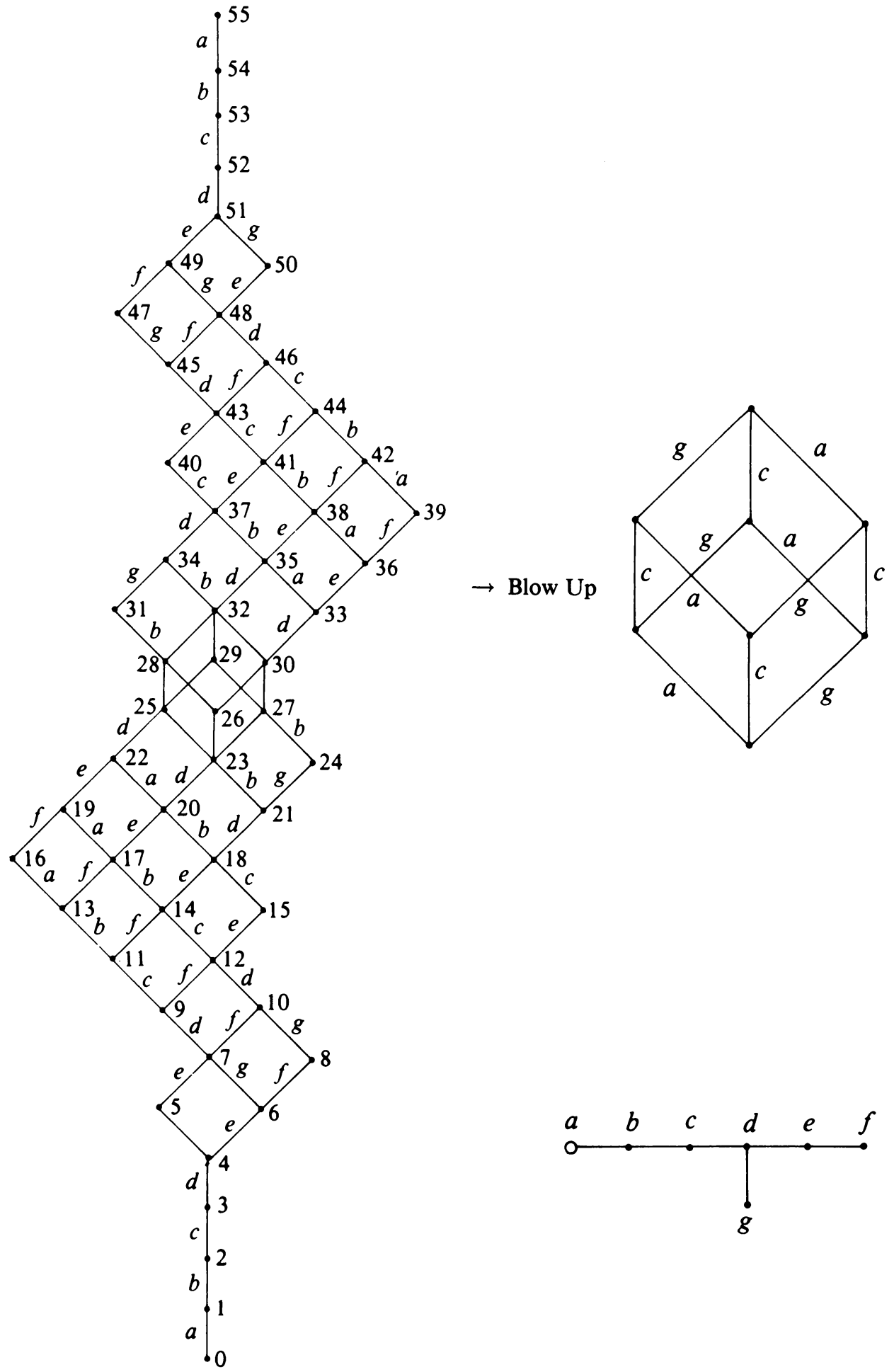

FIGURE 3.1. $\left(E_{7}, E_{6}\right)$ 

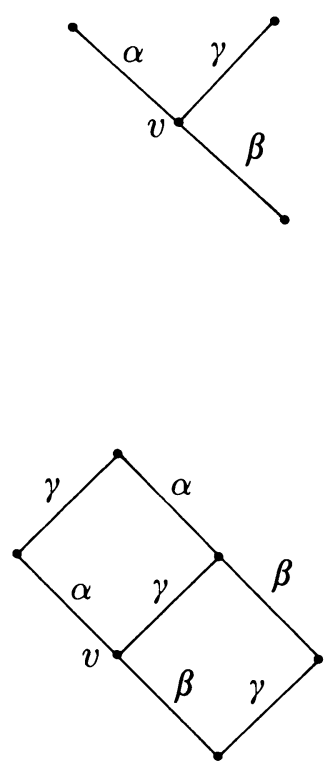

or

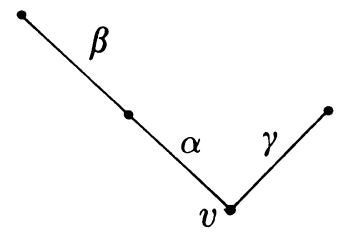

Respectively

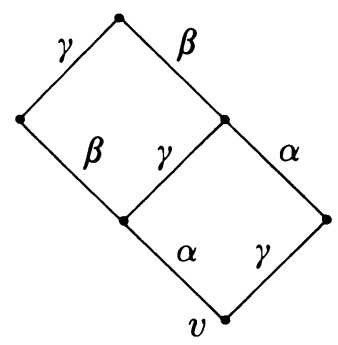

$N\left(w\left(\rho-\omega_{\beta}-\omega_{\gamma}\right)\right)$. The functor $\mu$ thus gives an equivalence between $\mathscr{O}_{\alpha \gamma}$ and $\mathscr{O}_{\beta \gamma}$ (cf. [4, Lemma 3.5]).

Lemma 3.5. Let $\alpha, \beta, \gamma$, and $\delta$ be any simple roots with $\alpha$ and $\gamma$ orthogonal and $\beta$ and $\delta$ orthogonal. Then the categories $\mathscr{O}_{\alpha \gamma}$ and $\mathscr{O}_{\beta \delta}$ are equivalent. Moreover, if $\mathscr{W}_{\alpha \gamma}=\left\{x_{1}, x_{2}\right\}$ with $x_{1}<x_{2}$ and $N_{i}=N\left(x_{i}\left(\rho-\omega_{\alpha}-\omega_{\gamma}\right)\right)$, $i=1,2$ then $N_{2}$ is projective and the projective cover of $N_{1}$ is a nonsplit extension of $N_{1}$ by $N_{2}$.

Proof. The first assertion follows easily from the previous lemma. So it suffices to analyze the case $\alpha=a$ and $\gamma=g$. Then $x_{1}=23$ and $x_{2}=26$ and $x_{2}=x_{1} s_{c}$. From Figure 3.1 we see that the category $\mathscr{O}\left(\rho-\omega_{a}-\omega_{c}-\omega_{g}\right)$ has only one simple module $A$ which must be a projective generalized Verma module. Tensoring from this cateogry out to $\mathscr{O}_{\text {ag }}$ (by a translation functor), $A$ must be sent to the projective cover of $N_{1}$ and this module is an extension of $N_{1}$ by $N_{2}$. This proves the lemma.

We now turn to the analysis of the semiregular categories. As in $\S 2$, all of the semiregular categories $\mathscr{O}_{\alpha}$ must be equivalent. As before, let $\tau_{\alpha \beta}: \mathscr{O}_{\alpha} \rightarrow \mathscr{O}_{\beta}$ be this equivalence whenever $\alpha$ and $\beta$ are adjacent simple roots. Then $\tau_{\alpha \beta}$ induces a bijection from $\mathscr{W}_{\alpha}$ to $\mathscr{W}_{\beta}$ which preserves the Bruhat order. From Figure 3.1 we obtain the Hasse diagrams of $\mathscr{W}_{f}$ and $\mathscr{W}_{a}$ as in (3.6).

Observe in particular that 16 is not less than 36 in the Bruhat order. As posets the sets $\mathscr{W}_{\alpha}, \alpha$ a simple root, are all isomorphic and we may enumerate all of these sets using the Hasse diagram in (3.7).

With this notation in hand we may describe the semiregular categories $\mathscr{O}_{\alpha}$. 

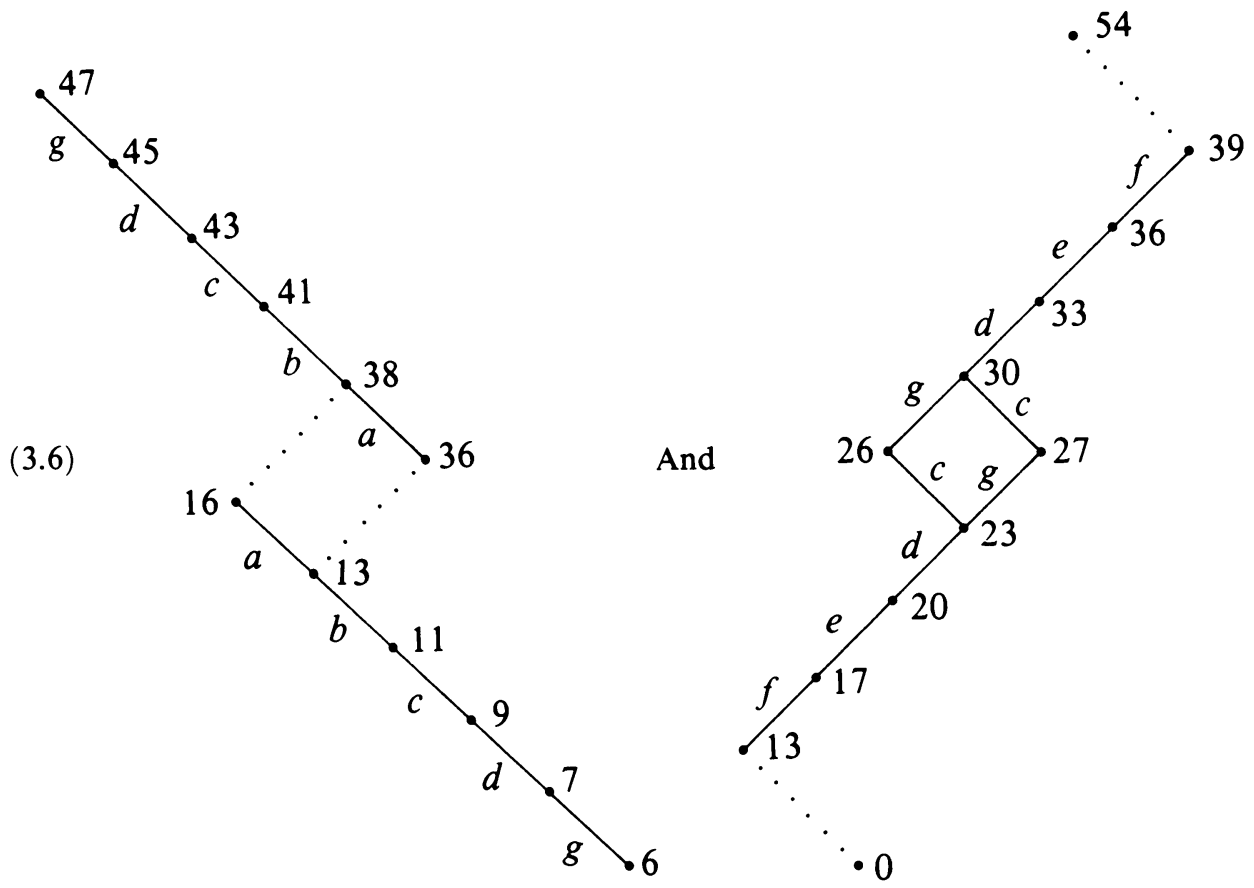

Proposition 3.8. Let $\alpha$ be a simple root. For $-5 \leq j \leq 5$ or $j=0^{\prime}$, let $N_{j}=N\left(v_{j}\left(\rho-\omega_{\alpha}\right)\right)$ and $L_{j}=L\left(v_{j}\left(\rho-\omega_{\alpha}\right)\right)$. Let $P_{j}$ be the projective cover of $N_{j}$ and $L_{j}$.

(i) For $0 \leq j \leq 4$ (resp. $\left.j=0^{\prime}\right), P_{j}$ is a nonsplit extension of $N_{j}$ by $N_{j+1}\left(\right.$ resp. $N_{0^{\prime}}$ by $\left.N_{1}\right) \cdot P_{5}=N_{5}$.

(ii) For $-5 \leq j \leq-2, P_{j}$ has a Verma flag of length 4 with flag factors $N_{j}, N_{j+1}, N_{-j}$, and $N_{-j-1}$.

(iii) $P_{-1}$ has a Verma flag of length 4 with flag factors $N_{1}, N_{0}, N_{0^{\prime}}$, and $N_{-1}$.

Proof. As mentioned before, all of the categories $\mathscr{O}_{\alpha}$ are equivalent. So to begin with we will assume that $\alpha$ is the simple root $f$ and identify the poset of (3.7) with the first poset of (3.6). It is well known that $P_{5}=N_{5}$. Let $j$ be in $\{-5,-4,-3,-2,0,1,2,3,4\}$. Then from (3.6) there is a unique simple root $\beta$ orthogonal to $f$ with $v_{j} \in \mathscr{W}_{f} \cap \mathscr{W}_{\beta}$. Let $\phi_{\beta}: \mathscr{O}_{f \beta} \rightarrow \mathscr{O}_{f}$ be the appropriate translation functor. Let $\widetilde{P}_{2}$ and $\widetilde{P}_{1}$ be the indecomposable projective modules in $\mathscr{O}_{f \beta}$ as described by (3.5) with $\widetilde{P}_{2}$ being a generalized Verma module. Then if $j \geq 0, \phi_{\beta}\left(\widetilde{P}_{2}\right)$ is the projective cover of $N_{j}$ and is the nonsplit extension of $N_{j}$ by $N_{j+1}$. If $j<0$ then $\phi_{\beta}\left(\widetilde{P}_{1}\right)$ is the projective cover of $N_{j}$ and since 


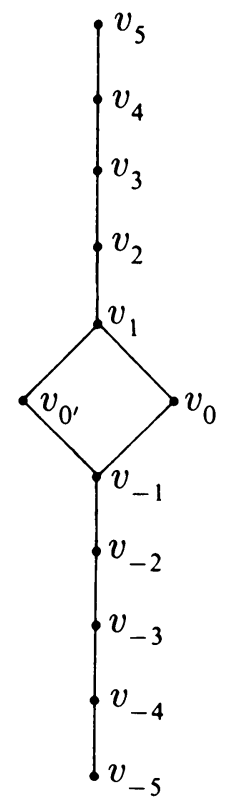

$\widetilde{P}_{1}$ has a Verma flag of length 2 with flag factors $\widetilde{P}_{2}$ and $\widetilde{P}_{1} / \widetilde{P}_{2}, \phi_{\beta}\left(\widetilde{P}_{2}\right)$ has a Verma flag of length 4 with flag factors $N_{j}, N_{j+1}, N_{-j}$, and $N_{-j-1}$.

It remains only to analyze the two points $v_{0^{\prime}}$ and $v_{-1}$. For these two cases we take $\alpha$ to be the simple root $a$ and identify (3.7) with the second poset in (3.6). Let $\phi_{g}: \mathscr{O}_{a g} \rightarrow \mathscr{O}_{a}$ be the appropriate translation functor and let $\widetilde{P}_{2}$ and $\widetilde{P}_{1}$ be the indecomposable projective modules in $\mathscr{O}_{a g}$ with $\widetilde{P}_{2}$ being a generalized Verma module. Then $\phi_{g}\left(\widetilde{P}_{2}\right)$ is the projective cover of $N_{0^{\prime}}$ and is an extension of $N_{0}$ by $N_{1}$. Similarly, $\phi_{g}\left(\widetilde{P}_{1}\right)$ is the projective cover of $N_{-1}$ and has flag factors $N_{-1}, N_{0}, N_{0^{\prime}}$, and $N_{1}$. This completes the proof of the proposition.

Proposition 3.9. Let $\alpha$ be a simple root and use the notation of (3.7) for $\mathscr{W}_{\alpha}$. For $v_{j} \in \mathscr{W}_{\alpha}$ let $N_{j}=N\left(v_{j}\left(\rho-\omega_{\alpha}\right)\right)$ and $L_{j}=L\left(v_{j}\left(\rho-\omega_{\alpha}\right)\right)$.

(i) For $0<j<t<6, \operatorname{Ext}^{k}\left(N_{-t}, L_{j}\right)= \begin{cases}\mathbf{C} \text { if } k=t+j \text { or } k=t-j \\ 0 & \text { otherwise. }\end{cases}$

(ii) For all indices $j$ and $l$ not covered by (i), $\operatorname{Ext}^{k}\left(N_{j}, L_{l}\right)=\left\{\begin{array}{cc}\mathbf{C} & \text { if } k=l-j \geq 0 \\ 0 & \text { otherwise. }\end{array}\right.$ (When either $j$ or $l$ equals $0^{\prime}$ then we interpret $0^{\prime}$ as the integer 0 in computing $k$.)

Proof. The same argument which proves Lemma 14.12 in [4] gives these formulas. What is involved is the information in Lemma 3.5 and the algebraic mapping cone (cf. 14.3 and 14.4 in [4]). 


\section{AsSOCIATED ROOT SYSTEMS}

All that remains to complete the program described in the introduction is to indicate the correspondence between the semiregular categories and certain subalgebras and subroot systems.

Fix a simple root $\alpha$ and let $w_{1}$ be the unique element of $\mathscr{W}_{\alpha}$ of minimal length. Here uniqueness is easily verified from Figures 2.1 and 3.1. Put $\mu_{1}=$ $w_{1}\left(\rho-\omega_{\alpha}\right)$ and $\gamma_{1}=-w_{1} \alpha$. Let $\mathscr{W}_{1}$ be the centralizer of $\gamma_{1}$ in $\mathscr{W}$ and $\Delta_{1}$ the root system of roots in $\Delta$ orthogonal to $\gamma_{1}$. Then $\mathscr{W}_{1}$ is the Weyl group of $\Delta_{1}$. Put $\Delta_{1, \underline{m}}=\Delta_{1} \cap \Delta_{\underline{m}}$ and $\Delta_{1}^{+}=w_{1} \Delta^{+} \cap \Delta_{1}$.

In the earlier sections we considered $\mathscr{W}_{\alpha}$ as a subset of the poset $\mathscr{W}^{m}$ given in Figures 2.1 and 3.1. These subsets become posets themselves if we include the edges given by the lines and dotted lines in (2.2), (2.3), and (3.6). Let $\mathscr{W}_{\alpha}$ also denote this poset.

Let $\mathscr{W}_{1, \underline{m}}$ denote the subgroup of $\mathscr{W}_{1}$ generated by the reflections $s_{\beta}$ with $\beta \in \Delta_{1, \underline{m}}$. Then, as with $\mathscr{W}$, we have $\mathscr{W}_{1}=\mathscr{W}_{1, \underline{m}} \cdot \mathscr{W}_{1}^{\underline{m}}$. We view $\mathscr{W}_{1}^{\underline{w}}$ as a poset associated to the pair $\left(\Delta_{1}, \Delta_{1, \underline{m}}\right)$ and the positive system $\Delta_{1}^{+}$.

\section{Lemma 4.1.}

(i) The pairs of root systems $\left(\Delta_{1}, \Delta_{1, m}\right)$ have types $\left(A_{5}, A_{4}\right)$ and $\left(D_{6}, D_{5}\right)$ in the cases of $E_{6}$ and $E_{7}$ respectively.

(ii) The posets $\mathscr{W}_{1}^{\underline{m}}$ and $\mathscr{W}_{\alpha}$ are isomorphic. An explicit isomorphism is given by $w \mapsto w w_{1}, w \in \mathscr{W}_{1}^{\underline{m}}$.

Proof. The type of the root system $\Delta_{1}$ is independent of the choice of $\alpha$. To determine $\Delta_{1}$ we give separate calculations for $E_{6}$ and for $E_{7}$.

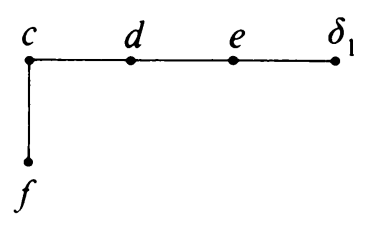

where $\delta_{1}=a+2 b+2 c+d+f$

For the case $E_{6}$ suppose $\alpha=a$. Then $\Delta_{1}$ contains the subroot system with Dynkin diagram (4.2). Now $|\Delta|=|\mathscr{W}| /\left|\mathscr{W}_{1}\right|$, and so $\left|\mathscr{W}_{1}\right|=6$ !. Thus, $\Delta_{1}$ is precisely the root system of type $A_{5}$ with Dynkin diagram given in (4.2).

Now return to the general setting for $E_{6}$, i.e. $\alpha$ is any simple root. Then $\mathscr{W}_{1, \underline{m}}$ is the stablizer in $\mathscr{W}_{\underline{m}}$ of $\gamma_{1}$. But $\mathscr{W}_{\underline{m}}$ acts transitively on $\Delta(\underline{u})$, and so $\left|\mathscr{W}_{1, \underline{m}}\right|=\left|\mathscr{W}_{\underline{m}}\right| /|\Delta(\underline{u})|=\underline{5}$ !. Now $\left(\Delta_{1}, \Delta_{1, \underline{m}}\right)$ corresponds to a Hermitian symmetric pair for $A_{5}$ with $\left|\mathscr{W}_{1, \underline{m}}\right|=5$ !. This implies that $\Delta_{1, \underline{m}}$ is of type $A_{4}$ and proves (i) for $E_{6}$.

For the case $E_{7}$ suppose $\alpha=a$. Then $\Delta_{1}$ contains the subroot system with Dynkin diagram (4.3). 


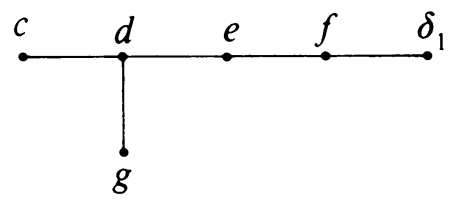

where $\delta_{1}=a+2 b+2 c+2 d+e+g$

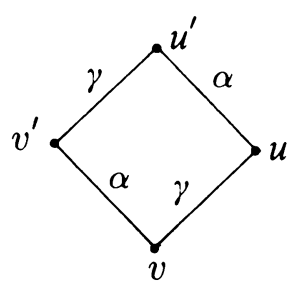

(4.4)

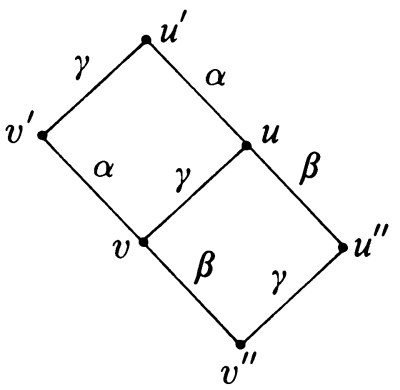

Respectively

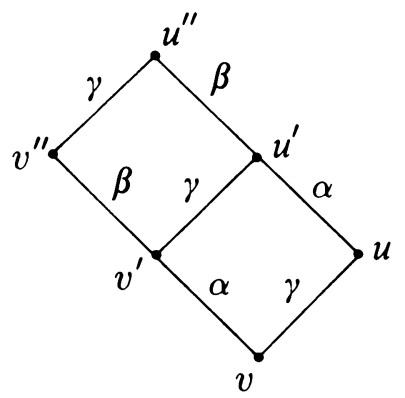

Arguing as above we find that $\left|\mathscr{W}_{1}\right|=6 ! 2^{5}$, and thus $\Delta_{1}$ is of type $D_{6}$ with Dynkin diagram given by (4.3).

Now suppose $\alpha$ is any simple root. The pair $\left(\Delta_{1}, \Delta_{1, \underline{m}}\right)$ corresponds to a Hermitian symmetric pair, and so $\Delta_{1, \underline{m}}$ is of type $D_{5}$ or $A_{5}$. However, arguing as above we see $\left|\mathscr{W}_{1, \underline{m}}\right|=\left|\mathscr{W}_{\underline{m}}\right| /|\Delta(\underline{u})|=5 ! 2^{4}$. So our pair is $\left(D_{6}, D_{5}\right)$, completing the proof of (i).

Suppose that the simple root $\gamma$ labels an edge in the poset $\mathscr{W}_{\alpha}$. Then in the poset $\mathscr{W}^{\underline{m}}$ we have a subdiagram (4.3.a) where $\gamma$ and $\alpha$ are orthogonal and $u$ and $v$ are in $\mathscr{W}_{\alpha}$. Now suppose $\beta$ is another simple root not orthogonal to $\alpha$. Since $u \alpha$ is in $\Delta\left(\underline{u}^{-}\right)$and $\underline{u}$ is Abelian, we conclude that either $(\mathrm{a}) u \beta$ is in $\Delta(\underline{u})$ or $(\mathrm{b}) u(\beta+\alpha)$ is in $\Delta\left(\underline{u}^{-}\right)$. Suppose $\beta$ and $\gamma$ are orthogonal. Then in cases (a) and (b) we have respectively the two cases of (4.4).

Next suppose $\beta$ and $\gamma$ are not orthogonal. First consider case $(a) ; u \beta$ is in $\Delta(\underline{u})$. Since $u \gamma$ is in $\Delta(\underline{u})$ and $v=u s_{\gamma}$ we have $v \beta=u s_{\gamma} \beta=u \beta+u \gamma$. Both $u \beta$ and $u \gamma$ are roots of $\underline{u}$, which is abelian. This contradiction eliminates this 


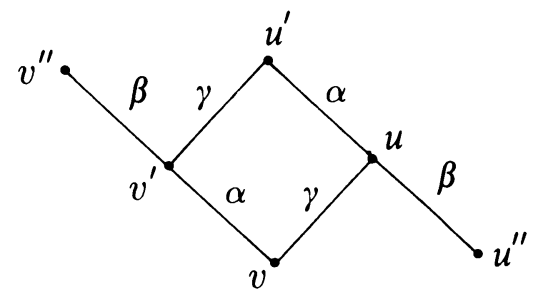

Respectively

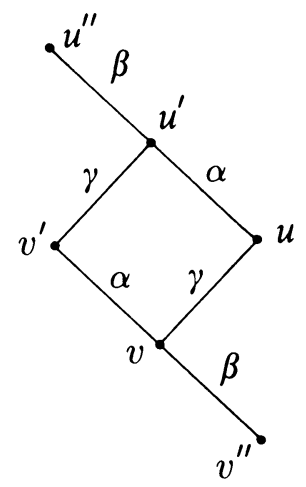

case. In case (b), $u \beta \in \Delta(\underline{m})$ and so $v \beta=u \beta+u \gamma \in \Delta(\underline{u})$. In this case we obtain the diagram in (4.5).

The functor $\tau_{\alpha \beta}$ carries $\mathscr{W}_{\alpha}$ to $\mathscr{W}_{\beta}$ and carries the pair $(u, v)$ to $\left(u^{\prime \prime}, v^{\prime \prime}\right)$, $\left(u^{\prime}, v^{\prime}\right),\left(u^{\prime \prime}, v^{\prime}\right)$, or $\left(u^{\prime}, v^{\prime \prime}\right)$ respectively in the four cases. In the first two cases right multiplication by $s_{\gamma}$ carries one element of the pair to the other. For the third and fourth cases $\delta=\alpha+\beta+\gamma$ is a root and the elements of the pairs are related by right multiplication by $s_{\delta}$. Also $\delta$ is orthogonal to $\beta$.

These facts prove that if $\mathscr{W}_{\alpha}$ lies in one $\mathscr{W}_{1}$-orbit for one choice of $\alpha$ then this holds for all $\alpha$. However, the verifications of this for $\alpha=a$ are easy with the dotted lines in (2.2) and (3.6) being given by the reflection $s_{\delta_{1}}$ with $\delta_{1}$ given as in (4.2) and (4.3). Now since $\left|\mathscr{W}_{1}^{\underline{m}}\right|$ equals $\left|\mathscr{W}_{\alpha}\right|, w \mapsto w w_{1}$ must carry $\mathscr{W}_{1}^{\underline{m}}$ to $\mathscr{W}_{\alpha}$. This completes the proof of (ii).

When we combine this lemma with the descriptions of the semiregular categories given in $\S 2$ and $\S 3$ we find that all of the results of the Memoir [4] hold here as well when we apply the constructions involving sets of orthogonal roots to the root system $\Delta_{1}$.

\section{REFERENCES}

1. B. Boe, T. J. Enright, and B. Shelton, Determination of the intertwining operators for holomorphically induced representations of Hermitian symmetric pairs, Pacific J. Math., 131 (1988), 39-50.

2. D. H. Collingwood, R. S. Irving, and B. Shelton, Filtrations on generalized Verma modules for Hermitian symmetric pairs, J. Reine Angew. Math., 383 (1988), 54-86.

3. T. J. Enright and B. Shelton, Decompositions in categories of highest weight modules, J. Algebra, 100 (1986), 380-402.

4. __ Categories of highest weight modules: Applications to classical Hermitian symmetric pairs, Mem. Amer. Math. Soc. 367, 1987. 
5. B. Shelton, Extensions between generalized Verma modules: The Hermitian symmetric cases, Math. Z. 197 (1988), 305-318.

6. D. Vogan, A generalized $\tau$-invariant for the primitive spectrum of a semisimple Lie algebra, Math. Ann. 242 (1979), 209-224.

Department of Mathematics, University of California at San Diego, la Jolla, CALIFORNIA 92093

Department of Mathematics, University of Oregon, Eugene, Oregon 97403 